\title{
REFLEXIVITY, FUNCTIONAL REFERENCE, AND MODULARITY: ALTERNATIVE TARGETS FOR LANGUAGE ORIGINS
}

\author{
TRAVIS LACROIX
}

\begin{abstract}
Researchers in language origins typically try to explain how compositional communication might evolve to bridge the gap between animal communication and natural language. However, as an explanatory target, compositionality has been shown to be problematic for a gradualist approach to the evolution of language. In this paper, I suggest that reflexivity provides an apt and plausible alternative target which does not succumb to the problems that compositionality faces. I further explain how proto-reflexivity, which depends upon functional reference, gives rise to complex communication systems via modular composition.
\end{abstract}

Keywords - reflexivity, language origins, explanatory targets, functional reference, modular composition, compositionality, animal communication

\section{INTRODUCTION}

Communication is ubiquitous in nature: every taxon that has been investigated displays some form of communication system (Kight et al., 2013). However, linguistic communication - i.e., natural language - is (or at least is often taken to be) unique to humans. This raises the question; how did language evolve? That is, how did rich linguistic communication systems like the ones we see in humans evolve out of simpler non-linguistic systems of communication? This is an inherently difficult question due to a lack of direct evidence - language does not fossilise, and we cannot observe the actual precursors of human language in, e.g., extinct hominin ancestors.

Nonetheless, work on language origins has blossomed in recent decades. New data, increasingly sophisticated techniques and technologies, and productive interdisciplinary research have helped foster the development of subtle models of language evolution. This is achieved using a multi-component approach to understand the mechanisms underlying language and how they might have evolved (Fitch,

Mila - Québec Artificial Intelligence Institute

DÉPARTEMENT D'informatique ET DE REChERChe OPÉRATIONNElle, Université de Montréal E-mail address: lacroixt@mila.quebec.

Date: Draft of June 15, 2020. 
2017). For example, comparative methods in evolutionary biology start by breaking down a complex trait into multiple subcomponent mechanisms or features (Fitch, 2017; Martinez, 2018). We can then examine the presence or absence of traits, in phylogenetic terms, to infer facts about whether some particular trait common to several species is a homologue or an analogue. Computer simulations further provide a concrete and explicit way to test hypotheses (Cangelosi and Parisi, 2002), furnishing a how-possibly explanation of the sort that is common in evolutionary biology (Resnik, 1991). However, the plausibility of these results requires figuring empirical evidence from relevant fields - in the case of language origins, this includes evidence from biology, linguistics, animal communication, neuroscience, and more.

The most common feature of natural language that is appealed to as a gapbridging explanatory target is compositionality (and related features like hierarchy and recursion). The idea is that if we could explain how compositional communication can evolve out of non-compositional communication, we would have taken great strides in explaining how language evolved. However, this is problematic insofar as (1) compositionality, in an evolutionary context, proffers asymmetric benefits for senders and receivers of signals, and researchers have not maintained adequate sensitivity to this role-asymmetry (LaCroix, 2020a); (2) there is no empirical evidence for proto-compositional communication as a precursor to natural language insofar as the oft-cited evidence is more likely homologous to human-level linguistic compositionality than analogous (LaCroix, 2019a); and (3) there is no gradualist explanation of compositionality, insofar as this is a binary property of language (Berwick and Chomsky, 2011; LaCroix, 2020b).

In this paper, I propose that reflexivity - the ability to use language to talk about language - provides an apt and plausible alternative explanatory target for language-origins research. I further explain how proto-reflexivity, which depends upon functional reference, gives rise to complex communication systems via modular composition. I argue that reflexivity does not succumb to the problems that compositionality faces since (1) role asymmetries are accounted for by the underlying mechanism of functional reference, (2) there exists empirical evidence of plausible precursors to reflexivity in nature, (3) the precursors of reflexivity are graded. Finally, reflexivity allows for rich compositional structures that have been shown to give rise to genuinely compositional syntax.

\section{Proto-reflexivity, functional reference, and their evolutionary PRECURSORS}

Communication is a unique evolutionary system in the following sense. Once a group of individuals has learned some simple communication convention, those learned behaviours may be used to influence future communicative behaviour, 
thereby affecting future communication conventions. This may give rise to a feedback loop, wherein more complex communication, in turn, is used to influence future communicative behaviours which are even more sophisticated.

When faced with a novel context, individuals can always learn a brand-new disposition from scratch. However, in some cases, it may be more advantageous or more efficient to utilise a pre-evolved disposition. When individuals take advantage of pre-evolved communicative dispositions to thereby influence future communication, this is a form of proto-reflexivity. Such an ability is an evolutionary precursor to the reflexivity of natural languages, wherein one can use language to talk about language.

Proto-reflexivity depends primarily upon functional reference, which has been the subject of much empirical and theoretical work in animal communication (Sievers and Gruber, 2016). Functional reference is so-called because it is meant to evoke the idea of reference in language without being equivalent to reference in the way that words refer. So, the ability to refer functionally is an evolutionary precursor to the ability to refer linguistically. Signals are functionally referential if they are 'elicited by a special class of stimuli and capable of causing behaviours adaptive to such stimuli in the absence of contextual cues' (Scarantino, 2013, 1006). ${ }^{1}$ They are therefore context-specific for the signaller to produce, and stimulus-independent for the receiver to understand. This can be defined formally, as in Definition 2.1.

Definition 2.1: (Strong) Functional Reference

A token of type $X$ functionally refers to a token of type $Y$ just in case the following two criteria are jointly satisfied:

(1) Production Criterion: $X \mathrm{~s}$ are reliably caused (only/mostly) by $Y \mathrm{~s}$;

(2) Perception Criterion: $X$ s presentations reliably cause responses adaptive to $Y \mathrm{~s}$ in the absence of $Y \mathrm{~s}$ and any other contextual cues.

For example, vervet monkey (Chlorocebus pygerythrus) alarm calls are suggested (Seyfarth et al., 1980) to be functionally referential since the presence of an eagle $(Y)$ reliably causes an eagle alarm call $(X)$, satisfying the production criterion. Furthermore, the presentation of an eagle alarm call $(X)$ reliably causes recipients to hide in the bush (an adaptive response to the presence of an eagle, $Y$ ), satisfying the perception criterion. Playback experiments suggest that these responses occur in the absence of other contextual cues.

Female Diana monkeys (Cercopithecus diana) elicit alarm calls upon viewing a predator first-hand and respond to alarm calls of male Diana monkeys by repeating the call. Zuberbühler et al. (1999) perform playback experiments of various pairs

${ }^{1}$ See also Macedonia and Evans (1993). 
of stimuli-a matching pair consists of an alarm call followed by the sound of the predator to which the call functionally refers; a mismatched pair consists of an alarm call followed by the sound of a predator to which the call does not functionally refer. In each case, pairs of stimuli are separated by five minutes of silence. In the experiment, the female monkeys displayed less concern upon hearing, e.g., the characteristic shriek of an eagle five minutes after the eagle alarm call-the former conveys no new information. However, they showed significant concern upon hearing a characteristic leopard growl five minutes after hearing the eagle alarm call. The conclusion is that alarm calls do not just serve to trigger (behaviourally or deterministically) an evasive response: individuals have an 'idea' - what Hurford (2007) terms a 'proto-concept' - of the relevant predator in mind for at least five minutes following the initial alarm call.

We might worry about the strength of Definition 2.1 since, for example, aggression signals may functionally refer to future aggressive behaviour, though it perhaps seems strange to say they are caused by it. We can weaken this by indexing to a context and replacing causation with correlation, as in Definition 2.2 (Scarantino, 2013):

Definition 2.2: (Weak) Functional Reference

A token of type $X$ in context $\mathbf{C}$ functionally refers to a token of type $Y$ just in case the following two criteria are jointly satisfied:

(1) Contextual Information Criterion: $X \mathrm{~S}$ in context $C$ are correlated with $Y$ s (weakly or strongly);

(2) Contextual Perception Criterion: $X$ s presentations in context $C$ reliably cause responses adaptive to $Y \mathrm{~s}$ in the absence of $Y \mathrm{~s}$.

This is information-theoretic because $X$ carries information about $Y$ just in case $X \mathrm{~s}$ and $Y \mathrm{~s}$ are correlated. ${ }^{2}$ The intuition is that the signal and the functional referent must correlate enough to make responding to the signal in ways that are adaptive to the referent evolutionarily advantageous.

Functional reference, and therefore proto-reflexivity, minimally requires several communicative precursors, including arbitrariness, specialisation, semanticity, discreteness, and displacement (Hockett, 1960). Arbitrariness requires that there is no 'natural' connection between a linguistic form and its meaning; this contrasts with iconic signals where there is a similarity between the form of a sign and its meaning - e.g., onomatopoeia in natural language. Specialisation requires that the signal produced is intended for communication, and not because of another

\footnotetext{
${ }^{2}$ This dovetails nicely with the role that information transfer plays in studies of animal communication (see Stegmann (2013), though cf. Dawkins and Krebs (1978)), as well as theoretical work in philosophy on meaning as informational content; see Skyrms (2010a,b).
} 
behaviour; this contrasts with cues, which are a byproduct of some other (noncommunicative) process - e.g., the presence of $\mathrm{CO}_{2}$ transfers information about the location of a mammal, though exhalation of $\mathrm{CO}_{2}$ did not evolve for this purpose. Semanticity requires that there is a relationship between a signal and its meaning. However, these three features of communication are early-evolving abilities that are common to mammals generally. Discreteness means that signals are perceived categorically, as opposed to continuously; this feature is present in primates generally. Finally, displacement is the ability to talk about things that are not present in the immediate environment.

Consider a situation where individuals coordinate upon a communication convention, like in a simple signalling game (Lewis, 1969; Skyrms, 2010a). In this case, the messages may functionally refer to the states of the world - as in the vervet monkey alarm call system. Now, suppose that this signalling situation occurs in a pre-evolved context. Suppose further that there is a novel context in which individuals must learn a new communication system. In some cases, the output of the novel signalling context may be an appropriate input for the pre-evolved signalling context (Barrett and Skyrms, 2017; LaCroix, 2020c); see Figure 1.

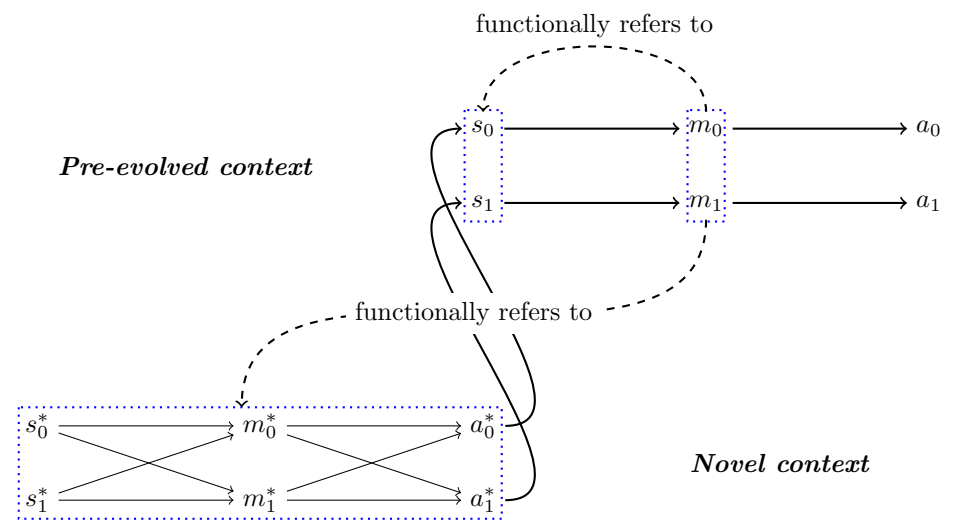

FiguRE 1

However, signals functionally refer to states in the pre-evolved context, and the states are just the output of the communication system in the novel context; so, messages come to functionally refer to the communication system itself, in a way that is proto-reflexive: they functionally refer to a communication context as a whole, rather than linguistic symbols themselves. In such a situation, discrete, arbitrary, and meaningful signals, which are specialised for communicative purposes, come to (functionally) refer to something abstract, in a sense, and so displaced from the immediate environment. 
How might such a property or ability evolve? This happens by way of modular composition and related processes. Various processes of this sort may include appropriation or template transfer, analogical reasoning, or genuine modular composition.

\section{Modular COMPOSItion AND RELATED PROCESSES}

3.1. Transfer (of) Learning. The simplest way of evolving new strategies from old strategies is appropriation. This process, minimally, requires the following. First, the agents must have evolved a disposition for a particular context. The agents then face a novel context, where the prior disposition just happens to be appropriatethough this may not be known at the outset. This novel context may be relevantly similar, but non-trivially distinct, from the original context. Appropriation then consists in applying the prior strategy to the novel context. It may be that the agent happens, by chance, to try something pre-evolved when faced with a novel context. The appropriateness of the pre-evolved strategy may determine a sufficiently beneficial reward such that, when faced with this same context again, the agent learns quickly (even by simple reinforcement) to perform the old action. This simple form of appropriation is sometimes called transfer (of) learning. ${ }^{3}$

This allows for flexibility of behaviour in problem-solving, via the ability to generalise learned rules to novel contexts. There is good evidence that many species of new- and old-world monkeys, as well as great apes, are capable of transfer; however, prosimians are not (Rumbaugh, 1970, 1971, 1995; Rumbaugh and Pate, 1984a,b; Bonte et al., 2014). One example of transfer learning in nonhuman animals is an extension of classification tasks, involving 'reversal learning'. Here, an animal is trained to associate a particular stimulus with a reward. Once the agent exhibits some degree of success, the relation between the stimulus and the reward reverses, so the agent must replace the prior association with the opposite association. If the animal can quickly reverse its associations, it is assumed that successful performance is based on a concept of oppositenEss. On the other hand, if the new association takes as long or longer to be learned, no such application of conceptual understanding may be attributed to the agent. ${ }^{4}$

Minimally, transfer learning requires only that an agent try prior strategies. Successful strategies may be learned via simple reinforcement, or they may be discovered via a more sophisticated trial-and-error. When salience is present - e.g., the physical properties of a new predator being saliently similar to an old predator - the

${ }^{3}$ See, e.g., Ellis (1965); Schunk (2004); Pugh and Bergin (2006); Hung (2013).

${ }^{4}$ Hurford (2007) argues that reversal learning experiments do not merely highlight an ability to apply the relation of OPPOSITENESS between a source and a target context; instead, the agent 'seems to be keeping its old mental representation (concept) of the general class of stimuli acquired in the first training regime and relating the new set to that acquired concept' (25). 
new strategy may be implemented immediately; however, this is a more sophisticated version of transfer learning, which requires a concept of analogical similarity.

3.2. Analogical Reasoning. The most common way of testing analogical reasoning ability is with a set of analogy problems known as relational matching-to-sample (RMTS) tasks. ${ }^{5}$ This experimental task involves showing the agent a sample set, which consists of two or more objects that are either identical or non-identical. The agent is then shown two comparison sets, which contain novel objects - one of which involves identity, and the other of which involves non-identity. To be successful, the agent must choose the comparison set which matches the sample set.

In this case, the analogy between various stimuli requires a concept of SAME versus Different. As with transfer learning, there is some evidence that nonhuman animals can utilise analogical reasoning. Despite prior belief to the contrary (Thompson and Oden, 2000), it has been shown experimentally that some apes (importantly, chimpanzees) can perform these tasks easily. Other apes and very few old-world monkeys can perform these tasks, but only after extensive training. In each case, symbolic training results in better performance, implying a relationship between cognition and linguistic ability. ${ }^{6}$

Noting and taking advantage of analogy is more cognitively complex than simple transfer. Increasing complexity again, we arrive at a full concept of modular composition.

3.3. Modular Composition. Finally, modular composition itself varies in complexity, but the most complex forms are supposed to be unique to humans and to depend upon language. Spelke (2003) suggests that humans and other animals are endowed with early-developing, core systems of knowledge, called 'modules'. However, these core systems are limited in several ways. First, they are domainspecific, since these modules represent only a subset of entities in the surroundings of the agent. Second, they are task-specific, since they inform only a subset of the repertoire of the agent's actions and cognitive processes. Third, they are (at least relatively) encapsulated, since there is a restriction on the flow of information into and out of a module. Finally, modules are (at least relatively) isolated from one another, since they do not readily combine (Spelke, 2003, 291). ${ }^{7}$

\footnotetext{
${ }^{5}$ See Skinner (1950); Blough (1959); Ferster (1960).

${ }^{6}$ See, e.g., Skinner (1950); Blough (1959); Ferster (1960); Fagot et al. (2001); Wasserman et al. (2001); Katz et al. (2002); Flemming et al. (2011).

${ }^{7}$ See also Fodor (1983, 1984, 2000); Sherry and Schacter (1987); Sperber (1994); Coltheart (1999); Sperber (2002); Carruthers (2002); Barrett and Kurzban (2006); Shettleworth (2012); Robbins (2017).
} 
Many core cognitive capacities that are available to (and were once thought to be unique to) humans are also available to nonhuman animals (Spelke, 2003). ${ }^{8}$ Therefore, humans, but also nonhuman animals, have early-developing core knowledge systems, which allow for a broad range of intelligent behaviour and cognitive capacities; and, in many cases, these same core systems enable nonhuman animals to outperform human infants in similar tasks. Thus, core systems alone do not account for uniquely human cognitive capacities. Spelke (2003) suggests that human cognitive capacities depend on core knowledge systems, which are shared by other animals, and on a uniquely human combinatorial ability for conjoining these representations to create new systems of knowledge. Furthermore, she suggests that the latter capacity is made possible by natural language, which provides the medium for combining the representations delivered by core knowledge systems (305). Specifically, it is the compositional nature of natural language, which gives rise to uniquely flexible human cognition, on her account.

The basic communicative abilities that give rise to human linguistic capacities are shared with many other species; however, the ability to produce and interpret recursive structures is uniquely human (Hauser et al., 2002). If we assume that the human capacity for language can be decomposed into a set of well-defined mechanisms that interact via interfaces, then we can begin to examine how such interfaces between individual components may 'hook up' in the first place. In essence, this is the concept of modular composition as it is described in Barrett and Skyrms (2017). Modular composition ties together explanations of complexity in communicative, cognitive, and social structures.

\section{REFlexivity AS AN EXPLANATORY TARGET}

Researchers typically propose evolutionary theories that explain how compositionality arose, moving from a one-word stage (simple signalling), to a two-word stage (combinatorial signalling), and eventually to (compositional) language. ${ }^{9}$ However, as was mentioned in the introduction, prioritising linguistic compositionality as an explanatory target gives rise to significant theoretical and practical problems.

The novel approach to the evolution of language suggested here prioritises reflexivity as an explanatory target. On this account, simple communicative capacities evolve alongside cognitive capacities. Signals may become functionally referential, referring to concrete objects in the world. Once individuals are able to make use of proto-concepts, they can refer to abstracta. Therefore, they can refer to communicative contexts, giving rise to proto-reflexivity. This ability means that they can

${ }^{8}$ See empirical work in Wynn (1992); Simon et al. (1995); Koechlin et al. (1998); de Walle et al. (2001); Feigenson et al. (2002). See Wynn (1998); Spelke (1998) for reviews of this literature. ${ }^{9}$ See, e.g., Bickerton (1990); Jackendoff (1999); Progovac (2015). 
influence future communicative behaviour via communication. Such capacities may evolve by modular composition and related processes. Furthermore, it has been demonstrated that reflexivity gives rise to functional composition (compositional syntax) as a byproduct of these processes (LaCroix, 2019b).

Several recent works in the signalling game literature have demonstrated that modular compositional processes, like the ones described here, are more efficient and more effective for evolving or learning communication conventions than learning novel dispositions from scratch, often by orders of magnitude (Barrett, 2016, 2017, 2020; Barrett and Skyrms, 2017; LaCroix, 2019b, 2020c; Barrett et al., 2020).

Furthermore, reflexivity does not succumb to the same problems that compositionality does, as an explanatory target. It was mentioned in the introduction that compositionality, as it is discussed in the literature, fails to maintain sensitivity to role-asymmetries between producers and interpreters of signals (LaCroix, 2020a); however, for reflexivity, this role-asymmetry is built-in via functional reference (Definitions $2.1 ; 2.2$ ), which accounts for these differences by definition. Furthermore, there are no empirical precursors to compositionality (LaCroix, 2019a), whereas the processes by which reflexivity evolves are supported by significant empirical evidence. Finally, compositionality is a binary property of language (Berwick and Chomsky, 2011), meaning that there is no gradualist explanation of the evolution of compositionality; in contrast, both reflexivity and the processes by which it might arise are graded notions. In non-reflexive functionally-referential systems, signals refer to states; in proto-reflexive functionally-referential systems, signals refer to communicative contexts; and in reflexive language, words refer to linguistic entities. So, reflexivity is graded, but the processes by which it arises are also gradedappropriation is simpler than analogical reasoning, which is simpler than modular composition.

Finally, compositionality is focused too internally on language and syntax itself, so explanations do not (or at least need not) take account of related cognitive and social mechanisms that are important factors in the evolution of language. On the other hand, reflexivity does. Therefore, there are significant practical and theoretical reasons to replace compositionality with reflexivity as an explanatory target for language origins research.

\section{REFERENCES}

Barrett, H. Clark and Robert Kurzban (2006). Modularity in Cognition: Framing the Debate. Psychological Review, 113(3): 628-647.

Barrett, Jeffrey (2016). On the Evolution of Truth. Erkenntnis, 81: 1323-1332.

Barrett, Jeffrey (2017). Truth and Probability in Evolutionary Games. Journal of Experimental and Theoretical Artificial Intelligence, 29(1): 219-225. 
Barrett, Jeffrey A. (2020). Self-assembling games and the evolution of salience. British Journal for the Philosophy of Science. Forthcoming.

Barrett, Jeffrey A. and Brian Skyrms (2017). Self-Assembling Games. The British Journal for the Philosophy of Science, 68(2): 329-353.

Barrett, Jeffrey A., Brian Skyrms, and Calvin Cochran (2020). Hierarchical Models for the Evolution of Compositional Language. Philosophy of Science. Forthcoming.

Berwick, Robert C. and Noam Chomsky (2011). The Biolinguistic Program: The Current State of its Development. In Sciullo, A. M. Di and C. Boeckx, editors, The Biolinguistic Enterprise: New Perspectives on the Evolution and Nature of the Human Language Faculty, pages 19-41. Oxford University Press, Oxford.

Bickerton, Derek (1990). Language and Species. University of Chicago Press, Chicago.

Blough, Donald S. (1959). Delayed Matching in the Pigeon. Journal of the Experimental Analysis of Behavior, 2(2): 151-160.

Bonte, Élodie, Caralyn Kemp, and Joël Fagot (2014). Age Effects on Transfer Index Performance and Executive Control in Baboons (Papio papio). Frontiers in Psychology, 5: 188.

Cangelosi, Angelo and Domenico Parisi (2002). Computer Simulation: A New Scientific Approach to the Study of Language Evolution. In Simulating the Evolution of Language, pages 3-28. Springer, London.

Carruthers, Peter (2002). The Cognitive Functions of Language. Behavioral and Brain Sciences, 25(3): 657-725.

Coltheart, Max (1999). Modularity and Cognition. Trends in Cognitive Sciences, 3(3): $115-120$.

Dawkins, Richard and John R. Krebs (1978). Animal signals: Information or Manipulation? In Krebs, J. R. and N. B. Davies, editors, Behavioural Ecology, pages 282-309. Blackwell Scientific Publications, Oxford.

de Walle, Gretchen A. Van, Susan Carey, and Meredith Prevor (2001). Bases for Object Individuation in Infancy: Evidence from Manual Search. Journal of Cognition and Development, 1(3): 249-280.

Ellis, Henry Carlton (1965). The Transfer of Learning. The Macmillan Company, New York.

Fagot, Jöel, Edward A. Wasserman, and Michael E. Young (2001). Discriminating the Relation Between Relations: The Role of Entropy in Abstract Conceptualization by Baboons (Papio papio) and Humans (Homo sapiens). Journal of Experimental Psychology: Animal Behavior Processes, 27(4): 316-328.

Feigenson, Lisa, Susan Carey, and Elizabeth S. Spelke (2002). Infants' Discrimination of Number vs. Continuous Extent. Cognitive Psychology, 44(1): 33-36. 
Ferster, Charles Bohris (1960). Intermittent Reinforcement of Matching to Sample in the Pigeon. Journal of the Experimental Analysis of Behavior, 3(3): 259-272.

Fitch, W. Tecumseh (2017). Empirical Approaches to the Study of Language Evolution. Psychonomic Bulletin 83 Review, 24(1): 3-33.

Flemming, Timothy M., Roger K. R. Thompson, Michael J. Beran, and David A. Washburn (2011). Analogical Reasoning and the Differential Outcome Effect: Transitory Bridging of the Conceptual Gap for Rhesus Monkeys (Macaca mulatta). Journal of Experimental Psychology: Animal Behavior Processes, 37(3): 353-360.

Fodor, Jerry (1983). The Modularity of Mind. MIT Press, Cambridge MA.

Fodor, Jerry (1984). Observation Reconsidered. Philosophy of Science, 51: 23-43.

Fodor, Jerry (2000). The Mind Doesn't Work That Way. MIT Press, Cambridge MA.

Hauser, Marc D., Noam Chomsky, and W. Tecumseh Fitch (2002). The Faculty of Language: What Is It, Who Has It, and How Did It Evolve? Science, 298: 15691579.

Hockett, Charles F. (1960). The Origin of Speech. Scientific American, 203: 88-111.

Hung, Woei (2013). Problem-Based Learning: A Learning Environment for Enhancing Learning Transfer. New Directions for Adult and Continuing Education, 137: 27-38.

Hurford, James R. (2007). Language in the Light of Evolution I: The Origins of Meaning. Oxford University Press, Oxford.

Jackendoff, Ray S. (1999). Possible Stages in the Evolution of the Language Capacity. Trends in Cognitive Sciences, 3: 272-279.

Katz, J. S., A. A. Wright, and J. Bachevalier (2002). Mechanisms of Same/Different Abstract-Concept Learning by Rhesus Monkeys (Macaca mulatta). Journal of Experimental Psychology: Animal Behavior Processes, 28(4): 358-368.

Kight, Caitlin R., John M. McNamara, David W. Stephens, and Sasha R. X. Dall (2013). Communication as information use: Insights from statistical decision theory. In Stegmann, Ulrich E., editor, Animal Communication Theory: Information and Influence, pages 89-112. Cambridge University Press, Cambridge.

Koechlin, Etienne, Stanislas Dehaene, and Jacques Mehler (1998). Numerical Transformations in Five-Month-Old Human Infants. Mathematical Cognition, 3(2): 89104.

LaCroix, Travis (2019a). Biology and compositionality: Empirical considerations for emergent-communication protocols.

LaCroix, Travis (2019b). Using logic to evolve more logic: Composing logical operators via self-assembly. British Journal for the Philosophy of Science. Forthcoming. 
LaCroix, Travis (2020a). Accounting for polysemy and role asymmetry in the evolution of compositional signals. unpublished manuscript.

LaCroix, Travis (2020b). Complex Signals: Reflexivity, Hierarchical Structure, and Modular Composition. PhD dissertation, University of California, Irvine.

LaCroix, Travis (2020c). The correction game or, how pre-evolved communicative dispositions might affect communicative dispositions. unpublished manuscript.

Lewis, David (2002/1969). Convention: A Philosophical Study. Blackwell, Oxford. Macedonia, Joseph M. and Christopher S. Evans (1993). Variation Among Mammalian Alarm Call Systems and the Problem of Meaning in Animal Signals. Ethology, 93: 177-197.

Martinez, Pedro (2018). The Comparative Method in Biology and the Essentialist Trap. Frontiers in Ecology and Evolution, 6(130): 1-5.

Progovac, Ljiljana (2015). Evolutionary Syntax. Oxford University Press, Oxford.

Pugh, Kevin J. and David A. Bergin (2006). Motivational Influences on Transfer. Educational Psychologist, 41(3): 147-160.

Resnik, David B. (1991). How-Possibly Explanations in Biology. Acta Biotheoretica, 39(2): 141-149.

Robbins, Philip (2017). Modularity of mind. In Zalta, Edward N., editor, The Stanford Encyclopedia of Philosophy. Metaphysics Research Lab, Stanford University, winter 2017 edition.

Rumbaugh, Duane M. (1970). Learning Skills of Anthropoids. In Rosenblum, L., editor, Primate Behavior: Developments in Field and Laboratory Research, pages 2-70. Aldine, New York.

Rumbaugh, Duane M. (1971). Evidence of Qualitative Differences in Learning Processes Among Primates. Journal of Comparative and Physiological Psychology, 76(2): 250-255.

Rumbaugh, Duane M. (1995). Primate Language and Cognition: Common Ground. Social Research, 62(3): 711-730.

Rumbaugh, Duane M. and James L. Pate (1984a). Primates' Learning by Levels. In Greenberg, G. and E. Tobach, editors, Behavioral Evolution and Integrative Levels, pages 221-240. Lawrence Erlbaum Associates, Hillsdale, NJ.

Rumbaugh, Duane M. and James L. Pate (1984b). The Evolution of Cognition in Primates: A Comparative Perspective. In Roitblat, H., T. G. Bever, and H. S. Terrace, editors, Animal Cognition, pages 569-587. Lawrence Erlbaum Associates, Hillsdale, NJ.

Scarantino, Andrea (2013). Animal Communication as Information-Mediated Influence. In Stegmann, Ulrich E., editor, Animal Communication Theory: Information and Influence, pages 63-87. Cambridge University Press, Cambridge. 
Schunk, Dale H. (2004). Learning Theories: An Educational Perspective. Pearson, Upper Saddle River, NJ, 4 edition.

Seyfarth, Robert M., Dorothy L. Cheney, and Peter Marler (1980). Vervet Monkey Alarm Calls: Semantic Communication in a Free-Ranging Primate. Animal Behaviour, 28(4): 1070-1094.

Sherry, David F. and Daniel L. Schacter (1987). The Evolution of Multiple Memory Systems. Psychological Review, 94(4): 439-454.

Shettleworth, Sara J. (2012). Modularity, Comparative Cognition and Human Uniqueness. Philosophical Transactions of the Royal Society B: Biological Sciences, 367(1603): 2794-2802.

Sievers, C. and T. Gruber (2016). Reference in human and non-human primate communication: What does it take to refer? Animal Cognition, 19(4): 759-768.

Simon, Tony J., Susan J. Hespos, and Philippe Rochat (1995). Do Infants Understand Simple Arithmetic? A Replication of Wynn (1992). Cognitive Development, 10(2): 253-269.

Skinner, Burrhus F. (1950). Are Theories of Learning Necessary? Psychological Review, 57: 193-216.

Skyrms, Brian (2010a). Signals: Evolution, Learning, E Information. Oxford University Press, Oxford.

Skyrms, Brian (2010b). The Flow of Information in Signaling Games. Philosophical Studies, 147(1): 155-165.

Spelke, Elizabeth S. (1998). Nativism, empiricism, and the origins of knowledge. Infant Behavior and Development, 21(2): 181-200.

Spelke, Elizabeth S. (2003). What Makes Us Smart? Core Knowledge and Natural Language. In Gentner, Dedre and Susan Goldin-Meadow, editors, Language in Mind: Advances in the Investigation of Language and Thought, pages 277-311. MIT Press, Cambridge, MA.

Sperber, Dan (1994). The Modularity of Thought and the Epidemiology of Representations. In Hirschfeld, L. A. and S. A. Gelman, editors, Mapping the Mind, pages 39-67. Cambridge University Press, Cambridge, MA.

Sperber, Dan (2002). In Defense of Massive Modularity. In Dupoux, I., editor, Language, Brain, and Cognitive Development, pages 47-57. MIT Press, Cambridge, MA.

Stegmann, Ulrich E. (2013). Animal Communication Theory: Information and Influence. Cambridge University Press, Cambridge.

Thompson, R. K. R. and D. L. Oden (2000). Categorical Perception and Conceptual Judgments by Non-Human Primates: the Paleological Monkey and the Analogical Ape. Cognitive Science: A Multidisciplinary Journal, 24(3): 363-396. 
Wasserman, Edward A., Michael E. Young, and Jöel Fagot (2001). Effects of Number of Items on the Baboon's Discrimination of Same from Different Visual Displays. Animal Cognition, 4(3-4): 163-170.

Wynn, Karen (1992). Addition and Subtraction by Human Infants. Nature, 358: $749-750$.

Wynn, Karen (1998). Psychological Foundations of Number: Numerical Competence in Human Infants. Trends in Cognitive Sciences, 2(8): 296-303.

Zuberbühler, Klaus, Dorothy L. Cheney, and Robert M. Seyfarth (1999). Conceptual Semantics in a Non-Human Primate. Journal of Comparative Psychology, 113(1): 33-42. 